\title{
Extraction of a dislocated leadless pacemaker in a patient with infective endocarditis and repeated endocardial and epicardial pacing system infections
}

\author{
Milos Taborsky ${ }^{\mathrm{a}}$, Tomas Skala ${ }^{\mathrm{a}}$, Martin Kocher ${ }^{\mathrm{b}}$, Marian Fedorco ${ }^{\mathrm{a}}$
}

\begin{abstract}
Background. Leadless pacemakers (PMs) were recently introduced to address the complication rate of standard PMs with transvenous leads.

Methods and Results. A 34-year old male with a history of intravenous substance abuse and a chronic type $\mathrm{C}$ hepatitis developed a complete atrioventricular block after cardiac surgery for infective endocarditis. Repeatedly, endo- and epi-cardially implanted PMs had to be explanted due to infection. A leadless MICRA pacemaker was successfully implanted with a dislocation into pulmonary artery several days after implantation. The PM was successfully retrieved using a single-loop retrieval snare guided by a steerable sheath. Subsequently, another Micra PM was successfully implanted with no further issues.

Conclusion. In the case of a Micra leadless pacemaker dislocation, a conventional gooseneck snare in combination with a steerable sheath can be used to retrieve the device, improving the overall safety of leadless pacemakers.
\end{abstract}

Key words: Micra, leadless, pacemaker, dislocation, extraction, gooseneck snare

Received: January 2, 2018; Accepted with revision: April 13, 2018; Available online: May 23, 2018 https://doi.org/10.5507/bp.2018.020

${ }^{a}$ Department of Internal Medicine I - Cardiology, Faculty of Medicine and Dentistry, Palacky University Olomouc and University Hospital Olomouc, I.P. Pavlova 6, Olomouc, Czech Republic

${ }^{b}$ Department of Radiological Methods, Faculty of Health Sciences, Palacky University Olomouc and Department of Radiology, University Hospital Olomouc, I.P. Pavlova 6, Olomouc, Czech Republic

Corresponding author: Tomas Skala, e-mail: tomasskala@gmail.com

\section{INTRODUCTION}

Standard pacemakers (PMs) with transvenous leads have a considerable complications rate. The short-term complication rate is approximately $9-10 \%$ (ref. ${ }^{1,2}$ ). The most significant complications are pocket hematoma, endocarditis, pneumothorax, lead dislodgement, venous obstruction, tricuspid regurgitation and cardiac tamponade ${ }^{3}$. Cardiac device infections occur in approximately $2.2 \%$ of device implantation and revision procedures, with $24 \%$ occurring more than two years after the device-related procedure ${ }^{4}$. Endovascular infection related to PM with transvenous leads implantation is associated with a mortality risk around 20\% $\left(\right.$ ref. $\left.^{5}\right)$. Transvenous leads can be affected with insulation breaks and lead body fracture that require another intervention with significant morbidity risk ${ }^{6}$. In many cases of PM infections and transvenous lead impairment, the stimulation system has to be extracted. Major vascular injury or cardiac perforation requiring emergency surgical or endovascular intervention during transvenous lead extraction is uncommon but carries significant inhospital mortality?

Leadless PMs were recently introduced to address some of these lead- and pocket-related complications. Two leadless pacing systems are currently clinically available: (A) the Nanostim Leadless Cardiac PM (LCP; St. Jude Medical), and (B) the Micra Transcatheter Pacing System (TPS; Medtronic) (ref. ${ }^{8,9}$ ).

\section{Case report}

A 34-year old male with a history of intravenous substance abuse and chronic type $\mathrm{C}$ hepatitis was admitted to hospital for infective endocarditis of the aortic and tricuspid valves. Infective endocarditis resulted in acute heart failure and the patient underwent cardiac surgery consisting of aortic valve replacement with bioprosthesis and tricuspid valve repair. An intracardiac abscess was treated by reconstruction of aortic bulbus and interventricular septum. An immediate postoperative period was complicated with pneumonia and respiratory failure. The patient was kept on a veno-venous extracorporeal membrane oxygenation system (ECMO) for six days.

A complete atrioventricular block (AVB) emerged right after surgery resulting in the need for temporary ventricular stimulation. While the AVB lasted, a transvenous PM was implanted after inflammatory markers levels decreased. One month after PM implantation, the patient presented with PM pocket infection. The pacing system had to be explanted. At that time, the patient was referred to our hospital. We extracted the whole pacing system and inserted a temporary pacing electrode. After prolonged antibiotics (ATB) treatment and decline in inflammatory marker levels, we performed a second transvenous PM implantation from the contralateral side. Unfortunately, 10 days after the implantation, PM pocket got infected again and the stimulation system had to be explanted for the second time. 
Since there was still a complete AVB, and since we had performed two extractions of the whole stimulation system, we decided to implant a leadless PM. Before the implantation, inflammatory markers levels were normal and focal infections were excluded. A leadless Micra pacemaker was implanted exactly according to the provided implantation protocol via right femoral vein with a stimulation threshold $0.9 \mathrm{~V}(0.40 \mathrm{~ms})$ after three stability tests. The stimulation threshold was considered adequate and the PM was released.

Three days after the implantation, the pacing threshold rose to $3.6 \mathrm{~V}(0.40 \mathrm{~ms})$ without a change in $\mathrm{PM}$ position or the detected signal. Due to the patient's dependence on stimulation, a screwed-in temporary pacing electrode was inserted as a safety measure to ensure stable stimulation. Fluoroscopy did not reveal any visible dislodgment of the pacemaker (Fig. 1.). We administered corticosteroid therapy aiming at improving the pacing threshold without apparent success. One week after the implantation, loss of leadless pacemaker capture was detected and fluoroscopy revealed an apparent dislocation of the PM into the left branch of the pulmonary artery. We tried to extract the dislocated leadless pacemaker. Since a dedicated extraction system was not available, a single-loop retrieval snare (COOK Medical, Bloomington, IN, USA) was inserted through right femoral vein. We have used a steerable sheath (Agilis, St. Jude Medical, St. Paul, MN, USA) that allowed us to manipulate the snare through the right ventricular outflow tract into the particular part of pulmonary artery where the PM was localized. The pacemaker was grasped and pulled back under fluoroscopic control and then surgically removed through the right femoral vein.

The patient was still dependent on the pacing and thus as a next possible step we decided to implant an epicardial

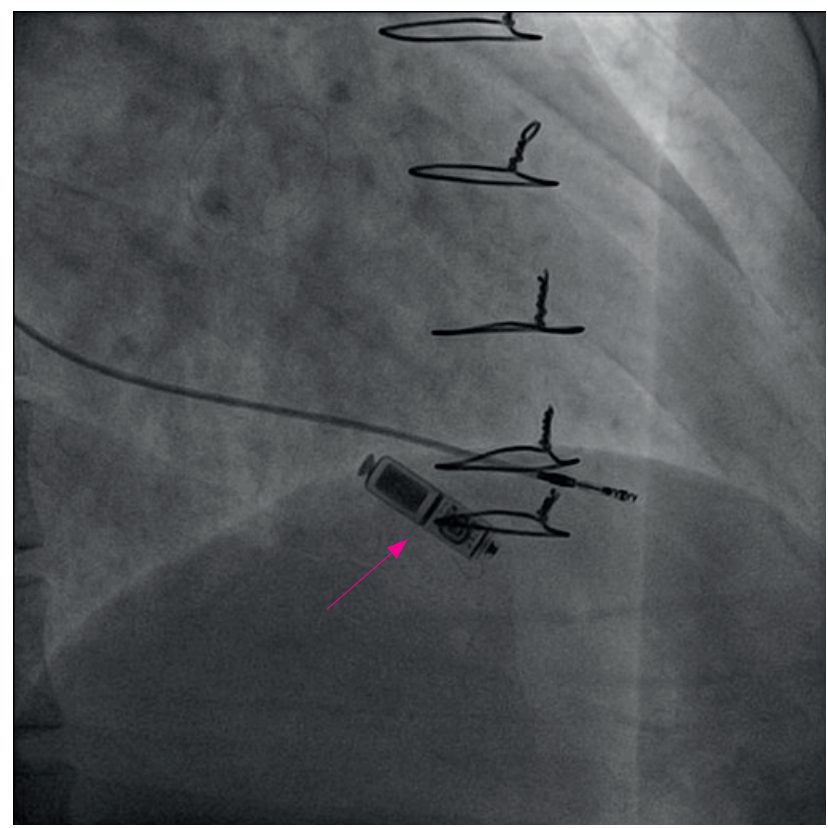

Fig. 1. Leadless pacemaker MICRA® (red arrow). Screwed-in temporary pacing electrode. pacing electrode. This was performed via anterolateral minithoracotomy with standard stimulation parameters. A permanent pacemaker was put under the left musculus rectus abdominis in the upper middle epigastrium and connected with the pacing electrode. After several days, there was a purulent secretion in thoracotomy. Microbiological analysis revealed Staphylococcus aureus as an infectious agent. The whole epicardial stimulation system had to be explanted again. Vacuum-assisted closure (VAC) therapy was needed to treat the non-healing chest wounds.

Subsequently, the patient had a spontaneous junctional rhythm of 42 beats per minute without bradycardia symptoms. This allowed us to treat him with prolonged combined antibiotics therapy under careful observation without any, not even a temporary, pacemaker lead inserted to minimize the possibility of persistent infection despite aggressive ATB therapy. During this treatment, the patient had a short worsening of bradycardia with syncope, so the need for a permanent pacemaker clearly remained and the patient could not have been left without a long-lasting PM stimulation. Thus, once the inflammatory markers declined and focal infection was excluded again, we decided to implant another pacemaker.

Since all pacemakers with transvenous leads had to be explanted, we decided to implant another leadless pacemaker Micra. The venous and epicardial access was limited. The left groin was not usable due to severe scarring after initial cardiosurgery and ECMO. The right groin was also severely scarred after surgical explantation of the first leadless PM. We decided to use suprapubic approach in the right groin. After several attempts in various parts of the right ventricle (RV), we were not able to find a spot with satisfactory stimulation parameters. Finally, two centimeters from the RV apex we found a spot with ac-

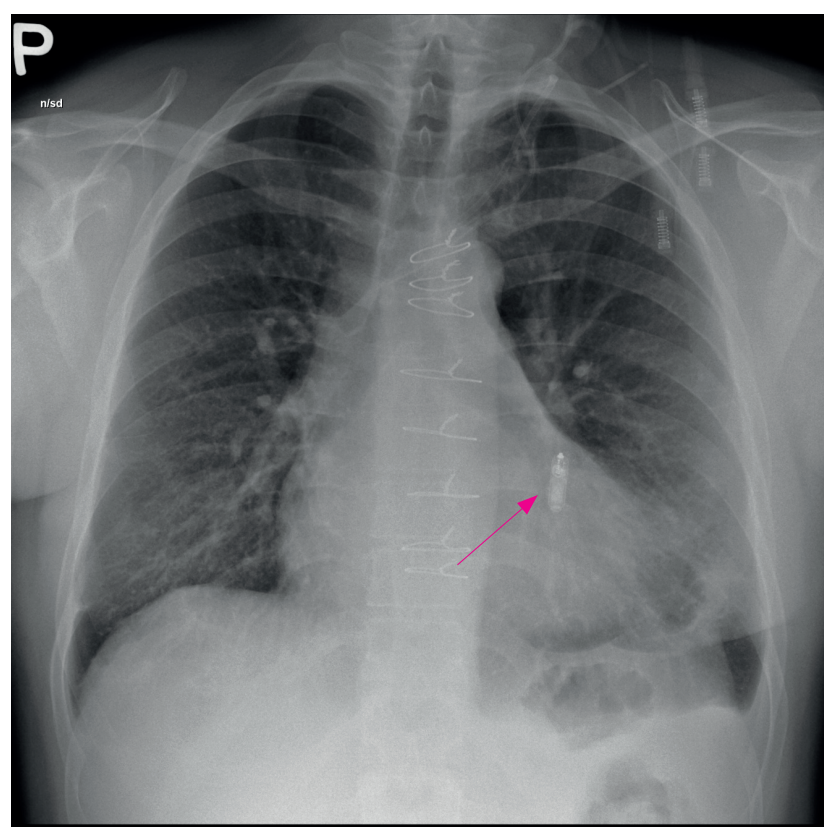

Fig. 2. Dislocated leadless pacemaker MICRA ${ }^{\circledR}$ (red arrow). 
ceptable pacing / sensing parameters and stability. Several measurements proved stable stimulation parameters even after the stability test. After many attempts, the best place found in the RV resulted in a stimulation threshold of $2.9 \mathrm{~V}(0.40 \mathrm{~ms})$. This threshold was high but there was no better spot in RV and since we ran out of options we decided to implant there.

The next day the PM parameters were as follows: threshold $2.25 \mathrm{~V}$, R-wave amplitude $8.4 \mathrm{mV}$, impedance $540 \mathrm{Ohm}$. These parameters remained stable from that moment on.

In the subsequent follow-up, the patient had no symptoms, he did not develop any sign of inflammation and the pacing parameters remained unchanged. The PM parameters are controlled remotely. The leadless PM was, the only viable option of stimulation in the long run.

\section{DISCUSSION}

Implantable transcatheter leadless PMs have several advantages over conventional pacing systems. Obviously, the use of these devices offers a reduction in pocket- and transvenous-lead- related issues. Nevertheless, the risk of a leadless pacemaker dislocation has to be considered. The

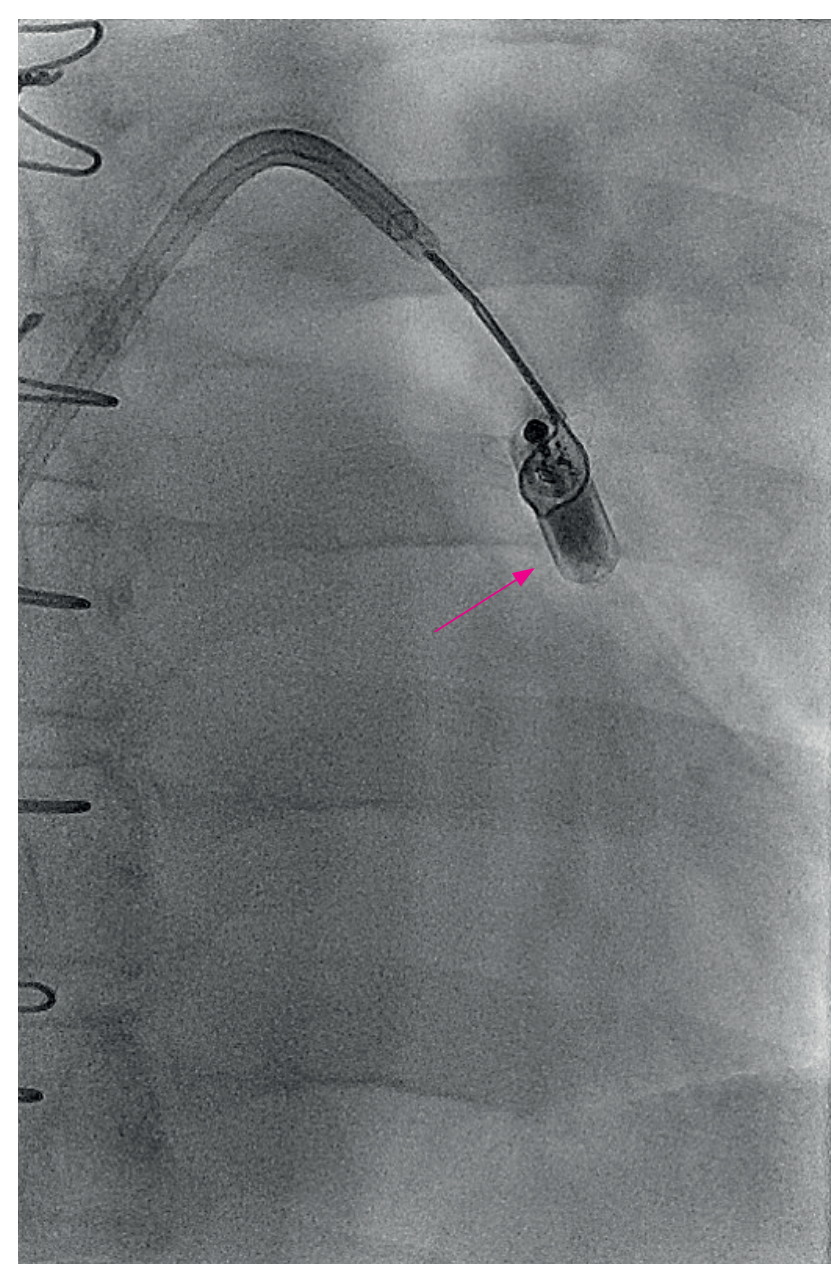

Fig. 3. Extraction of leadless pacemaker MICRA ${ }^{\circledR}$ using singleloop retrieval snare (red arrow). risk is mostly related to the implant procedure: cardiac perforation, device dislocation, and femoral vascular access site complications ${ }^{10}$. However, in cases such as the presented one, a leadless PM can be a viable option of stimulation and sometimes even the only one.

The stimulation threshold in Micra patients can change over time. Pacing threshold in most Micra patients with elevated thresholds decrease after implant. According to the Results from the Micra Transcatheter Pacing System Global Clinical Trial, stimulation thresholds of Micra patients with high $(>1.0 \mathrm{~V})$ and very high $(>1.5 \mathrm{~V})$ thresholds decreased significantly by 1 month, with $87 \%$ and $85 \%$ having 6 -month thresholds lower than the implant value. However, when the capture threshold at implant was $>2 \mathrm{~V}$, only $18.2 \%$ had a threshold of $\leq 1 \mathrm{~V}$ at 6 months and $45.5 \%$ had a capture threshold of $>2 \mathrm{~V}$. Micra device repositioning thus may not be necessary if the pacing threshold is $\leq 2 \mathrm{~V}$ ( ref. $^{11}$ ).

The delivery system allows for the PM to be repositioned several times during the implantation. After de-

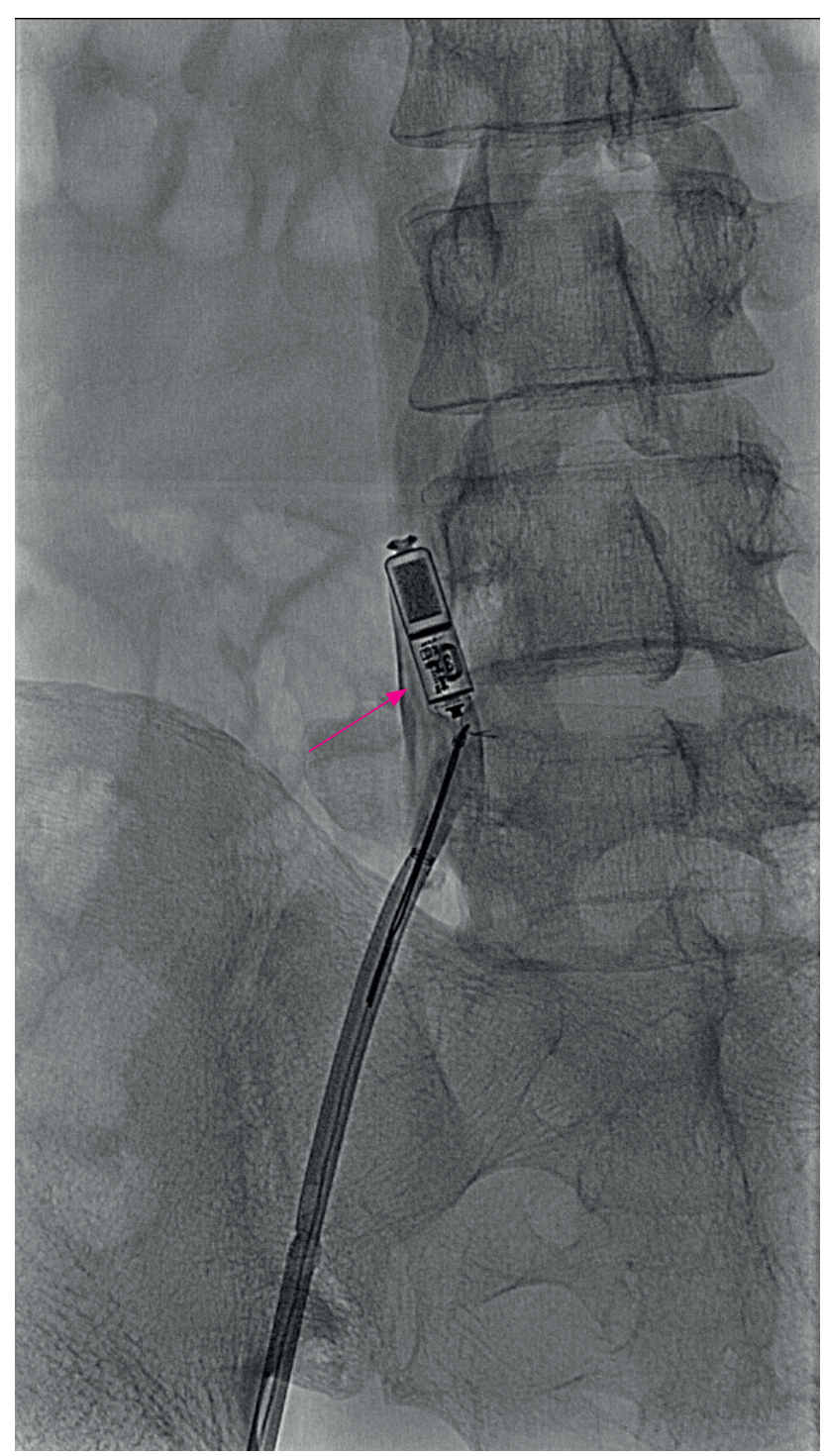

Fig. 4. Extraction of leadless pacemaker MICRA ${ }^{\circledR}$ using singleloop retrieval snare (red arrow). 
livery of the PM the recapture is more difficult but it is feasible using available foreign body removal snares even several weeks after implantation ${ }^{12}$.

In the presented case, in the first leadless PM implantation the stimulation threshold was $0.9 \mathrm{~V}$ after stability test. It was considered adequate and the PM was released. However, in three days a routine inspection of stimulation parameters after implantation revealed the stimulation threshold increase to $3.6 \mathrm{~V}$ without a change in PM position or the detected signal. To the best of our knowledge, such an impairment of stimulation parameters is extremely rare and the only plausible explanation is a PM micro-dislodgement, although the PM is actively fixed using the nitinol tines. A macro-dislodgement was observed after a few days and thus has to be expected in such a case and a temporary pacing electrode insertion should be considered.

In the FDA investigational device exemption (IDE) Micra TPS Trial (Micra Transcatheter Pacing Study, $\mathrm{n}=$ 745), an increased pacing threshold was documented in $0.3 \%$ of patients. No device dislodgements were observed, the implant success rate was excellent (99.2\%) and the major complication rate through 12 months post implant was low (4\%) (ref. ${ }^{9}$ ). According to the real-world data from a worldwide Post-Approval Micra Registry, device dislodgement was documented in only 1 of 795 implantations $(0.13 \%)$ so far. Performance of the Micra transcatheter pacemaker in a real-world setting demonstrates a high rate $(99.6 \%)$ of implant success and low rate $(1.51 \%)$ of major complications through 30 days post implant ${ }^{9}$. Remarkably, across a combined 1521 patients from both the IDE and registry studies, only 1 macrodislodgement has been reported so far $(0.066 \%)$ ( ref. $\left.^{13}\right)$. So the presented case is an extremely rare complication.

This does not mean that leadless PMs are more prone to lead dislodgement than standard transvenous PM systems. In the FOLLOWPACE study $(n=1517)$, lead dislodgement in transvenous PM systems was reported at a rate of $3.3 \%$ within 2 months of implant ${ }^{1}$. When the patients from the Pre-market Micra Transcatheter Pacing Study and the Micra Transcatheter Pacing System Continued Access Study (TPS; N = 989) were analyzed and compared directly with patients with transvenous pacemakers (TVP; $n=2667$ ) the actual rate for revision at 24 months postimplant was $1.4 \%$ for the TPS group (11 revisions in 10 patients), $75 \%$ (95\% confidence interval $53 \%-87 \%$; $P<0.001)$ lower than the $5.3 \%$ for the TVP group (123 revisions in 117 patients) (ref. ${ }^{14}$ ).

In the first Micra implantation, we proceeded precisely according to the company recommendations and the stimulation threshold was acceptable after three stability tests and device release. We were not able to find an operator-based fault leading to the dislodgement. This is supported by the fact, that in the Micra study, there was no evidence of a relationship between operator experience and major complications ${ }^{15}$. Moreover, the implanting operator in this case is an extremely experienced one, well beyond the learning curve. The precise reason for the PM dislodgment in the presented case is unknown. We can only speculate, that in a patient with a long history of intravenous substance abuse and infective endocarditis, the right ventricle trabeculae are remodeled resulting in difficulties in stable fixation of leadless PM with passive fixation.

Since device dislodgment is possible, the leadless PMs retrievability is an important feature. The Nanostim Leadless Cardiac PM has a dedicated steerable retrieval catheter $^{10}$. The Micra Transcatheter Pacing System does not but use of a conventional gooseneck snare was reported to be feasible ${ }^{12}$. We also solved this rare complication using a conventional gooseneck snare. This possibility of device retrieval is really important for the overall safety of the Micra leadless stimulation system.

In second leadless PM implantation, the stimulation parameters were suboptimal from the beginning. After many attempts, the best place that could be found in RV resulted in a stimulation threshold of $2.9 \mathrm{~V}$ and we were not able to find a better spot. In the Post-Approval Micra Registry population, 97\% of patients had an implant threshold of $<2 \mathrm{~V}$, and $87.2 \% \leq 1 \mathrm{~V}$, similar to what was reported for the IDE study ${ }^{9,13}$. The threshold in our case was very high, but since we ran out of options, we decided to implant the PM at the spot with the best, though suboptimal, parameters. Fortunately the stimulation threshold slightly decreased the next day and no dislodgment occurred in the follow-up.

The patient has been well so far. The leadless PM was the only possibility and literally saved his life.

\section{CONCLUSION}

Leadless pacemakers were recently introduced to address lead- and pocket-related complications. In cases such as the presented one, a leadless pacemaker can be a viable option for stimulation and sometimes even the only one. The risk of a leadless pacemaker dislocation is extremely small but it has to be considered. In the case of a Micra pacemaker dislodgment, a conventional steerable sheath in combination with a gooseneck snare can be used to retrieve the device, improving the overall safety of leadless pacemakers.

Author contributions: All authors contributed to the management and presentation of the case equally.

Conflict of interest statement: None declared

\section{REFERENCES}

1. Udo EO, Zuithoff NP, van Hemel NM, de Cock CC, Hendriks T, Doevendans PA, Moons KG. Incidence and predictors of short- and long-term complications in pacemaker therapy: the FOLLOWPACE study. Heart Rhythm 2012;9:728-35.

2. Kirkfeldt RE, Johansen JB, Nohr EA, Jorgensen OD, Nielsen JC. Complications after cardiac implantable electronic device implantations: an analysis of a complete, nationwide cohort in Denmark. Eur Heart J 2014;35(18):1186-94.

3. Harcombe AA, Newell SA, Ludman PF, Wistow TE, Sharples LD, Schofield PM, Stone DL, Shapiro LM, Cole T, Petch MC. Late compli- 
cations following permanent pacemaker implantation or elective unit replacement. Heart 1998;80:240-4.

4. Lekkerkerker JC, van Nieuwkoop C, Trines SA, van der Bom JG, Bernards A, van de Velde ET, Bootsma M, Zeppenfeld K, Jukema JW, Borleffs JW, Schalij MJ, van Erven L. Risk factors and time delay associated with cardiac device infections: Leiden device registry. Heart 2009;95:715-20.

5. Tarakji KG, Wazni OM, Harb S, Hsu A, Saliba W, Wilkoff BL. Risk factors for 1-year mortality among patients with cardiac implantable electronic device infection undergoing transvenous lead extraction: the impact of the infection type and the presence of vegetation on survival. Europace 2014;16:1490-5.

6. Hauser RG, Hayes DL, Kallinen LM, Cannom DS, Epstein AE, Almquist AK, Song SL, Tyers GF, Vlay SC, Irwin M. Clinical experience with pacemaker pulse generators and transvenous leads: an 8-year prospective multicenter study. Heart Rhythm 2007;4:154-60.

7. Brunner MP, Cronin EM, Wazni O, Baranowski B, Saliba WI, Sabik JF, Lindsay BD, Wilkoff BL, Tarakji KG. Outcomes of patients requiring emergent surgical or endovascular intervention for catastrophic complications during transvenous lead extraction. Heart Rhythm 2014;11:419-25.

8. Reddy VY, Exner DV, Cantillon DJ, Doshi R, Bunch TJ, Tomassoni GF, Plunkitt K. Percutaneous implantation of an entirely intracardiac leadless pacemaker. N Engl J Med 2015;373(12),1125-35.

9. Reynolds D, Duray GZ, Omar R, Soejima K, Neuzil P, Zhang S, Narasimhan C, Steinwender C, Brugada J, Lloyd M, Roberts PR, Sag V, Hummel J, Bongiorni MG, Knops RE, Ellis CR, Gornick CC, Bernabe MA, Laager V, Stromberg K, Williams ER, Hudnall JH, Ritter P, Micra
Transcatheter Pacing Study Group. A leadless intracardiac transcatheter pacing system. N Engl J Med 2016;374:533-41.

10. Tjong FVY, Reddy VY. Permanent Leadless Cardiac Pacemaker Therapy. Circulation 2017; 135(15):1458-70.

11. Piccini JP, Stromberg K, Jackson KP, Laager V, Duray GZ, El-Chami M, Ellis CR, Hummel J, Jones R, Kowal RC, Narasimhan C, Omar R, Ritter P, Roberts PR, Soejima K, Zhang S, Reynolds D. Long-term outcomes in leadless Micra transcatheter pacemakers with elevated thresholds at implantation: Results from the Micra Transcatheter Pacing System Global Clinical Trial. Heart Rhythm 2017;14(5):685-91.

12. Karim S, Abdelmessih M, Marieb M, Reiner E, Grubman E. Extraction of a Micra Transcatheter Pacing System: first-in-human experience. Heart Rhythm Case Reports 2016;2(1):60.

13. Roberts PR, Clementy N, Al Samadi F, Garweg C, Martinez-Sande JL, lacopino S, Johansen JB, Prat XV, Kowal RC, Klug D, Mont L, Steffel $\mathrm{J}, \mathrm{Li}$ S, Osch DV, El-Chami LF. A leadless pacemaker in the real-world setting: The Micra Transcatheter Pacing System Post-Approval Registry. Heart Rhythm 2017; 14(9):1375-1379. doi: 10.1016/j. hrthm.2017.05.017

14. Grubman $E$, Ritter $P$, Ellis $C R$, Giocondo $M$, Augostini $R$, Neuzil $P$, Ravindran B, Patel AM, Omdahl P, Pieper K, Stromberg K, Hudnall $J H$, Reynolds D. To retrieve, or not to retrieve: System revisions with the Micra transcatheter pacemaker. Heart Rhythm 2017;14(12):18016. doi: 10.1016/j.hrthm.2017.07.015

15. Kowal R, Soejima K, Ritter P, Duray GZ, Hudhall JH, Stromberg K, Reynolds D. Relationship between operator experience and procedure outcomes with the Micra transcatheter leadless pacing system. Heart Rhythm 2016;13:S169. 